Kanagasabai Balamurugan, Linda Koehler, Jan-Niklas Dürig, Ute Hempel, Jörg Rademann, Vera Hintze and M. Teresa Pisabarro*

\title{
Structural insights into the modulation of PDGF/ PDGFR- $\beta$ complexation by hyaluronan derivatives
}

https://doi.org/10.1515/hsz-2021-0173

Received February 26, 2021; accepted June 18, 2021;

published online July 20, 2021

\begin{abstract}
Angiogenesis is an important physiological process playing a crucial role in wound healing and cancer progression. Vascular endothelial growth factor (VEGF) and platelet derived growth factor (PDGF) are key players in angiogenesis. Based on previous findings regarding the modulation of VEGF activity by glycosaminoglycans (GAG), here we explore the interaction of hyaluronan (HA)-based GAG with PDGF and its receptor PDGFR- $\beta$ by applying molecular modeling and dynamics simulations in combination with surface plasmon resonance (SPR). Computational analysis on the interaction of oligo-hyaluronan derivatives with different sulfation pattern and functionalization shows that these GAG interact with PDGF in relevant regions for receptor recognition, and that high sulfation as well as modification with the TAMRA group convey stronger binding. On the other hand, the studied oligohyaluronan derivatives are predicted to scarcely recognize PDGFR- $\beta$. SPR results are in line with the computational predictions regarding the binding pattern of HA tetrasaccharide (HA4) derivatives to PDGF and PDGFR- $\beta$. Furthermore, our experimental results also show that the complexation of PDGF to PDGFR- $\beta$ can be modulated by HA4 derivatives. The results
\end{abstract}

Kanagasabai Balamurugan and Linda Koehler contributed equally.

*Corresponding author: M. Teresa Pisabarro, Structural

Bioinformatics, BIOTEC Technische Universität Dresden, Tatzberg 47 51, D-01307 Dresden, Germany, E-mail: maria_teresa.pisabarro@tudresden.de

Kanagasabai Balamurugan, Structural Bioinformatics, BIOTEC Technische Universität Dresden, Tatzberg 47-51, D-01307 Dresden, Germany

Linda Koehler and Vera Hintze, Institute of Materials Science, Max Bergmann Center of Biomaterials, Technische Universität Dresden, Budapester Str. 27, D-01069 Dresden, Germany. https://orcid.org/ 0000-0002-5611-9903 (V. Hintze)

Jan-Niklas Dürig and Jörg Rademann, Medicinal Chemistry Department, Institute of Pharmacy, Freie Universität Berlin, KöniginLuise-Straße 2+4, D-14195 Berlin, Germany. https://orcid.org/00000001-6678-3165 (J. Rademann)

Ute Hempel, Institute of Physiological Chemistry, Carl Gustav Carus Faculty of Medicine, Technische Universität Dresden, Fiedlerstraße 42, D-01307 Dresden, Germany found open the path for considering HA4 derivatives as potential candidates to be exploited for modulation of the PDGF/ PDGFR- $\beta$ signaling system in angiogenesis and related disease conditions.

Keywords: angiogenesis; extracellular matrix (ECM); glycosaminoglycans (GAG); hyaluronan (HA); platelet derived growth factor (PDGF); vascular endothelial growth factor (VEGF).

\section{Introduction}

Platelet-derived growth factors (PDGF) are important proteins acting as potent mitogens for connective tissue cells such as fibroblasts and smooth muscle cells and, in turn, regulating processes such as embryonic development and tissue regeneration (Kohler and Lipton 1974; Ross et al. 1974). PDGF exists in four isoforms, namely PDGF-A, -B, -C and -D, forming homodimers and heterodimers (i.e. PDGF-AA, - $\mathrm{BB}$, $-\mathrm{CC}$, -DD and -AB). PDGF interacts with its corresponding receptors PDGFR- $\alpha$ and PDGFR- $\beta$ to carry out distinct functions. PDGFR- $\alpha$ is involved in the signaling controlling gastrulation and in the development of various organs including intestine, lung, skin, kidney and testis; whereas PDGFR- $\beta$ signaling is well established in the process of early hematopoiesis and blood vessel formation (Andrae et al. 2008). PDGF-BB is a ligand for PDGFR- $\beta$, which is relevant in angiogenesis. Enhanced PDGF/PDGFR signaling is also observed in a variety of disease conditions such as cancer, pulmonary fibrosis and atherosclerosis (Ostman 2004). Due to the significance of the signaling of the PDGF/PDGFR system, its inhibition has become an attractive strategy, for instance, in anti-cancer therapy (Gialeli et al. 2014).

PDGF and vascular endothelial growth factors (VEGF) are related structurally and functionally. Structurally, both consist of an evolutionarily conserved cysteine-knot fold of around 100 amino acids, which is involved in dimerization (i.e. disulfide-linked dimer form) and binding to the corresponding receptor as shown in Figure S1 (Fredriksson et al. 2004; Shim et al. 2010). Functionally, both growth factors are involved in vascular formation or angiogenesis (Banfi et al. 2012). Since PDGFs and VEGFs are of common origin, their respective receptors are also related (PDGFRs 
and VEGFRs, made up of 5 and 7 immunoglobulin domains, respectively), and the structural features essential for the protein ligand/receptor recognition complex are also perceived to be analogous (Schlessinger 2000).

The combined inhibition of PDGF and VEGF signaling has emerged as a potential route for suppressing angiogenesis in tumor progression (Erber et al. 2004). It was recently found that the transition between normal and aberrant angiogenesis was not exclusively dependent on the VEGF dose but a delicate balance between the endothelial stimulation by VEGF and pericyte recruitment by PDGF-BB (Gianni-Barrera et al. 2018). PDGF-BB has been recently found to play a key role in pathological angiogenesis in osteoarthritis ( $\mathrm{Su}$ et al. 2020). Several strategies have been used to block PDGF/PDGFR signaling such as neutralizing antibodies for ligands and receptors, N-terminal processing-deficient PDGFs, aptamers, soluble receptors without the kinase domain and by natural cyclopeptide Destruxin A5 (Andrae et al. 2008; Kuai et al. 2015; Siegfried et al. 2005; Wang et al. 2016).

Based on our previous studies on the modulation of angiogenesis by the interaction of glycosaminoglycans (GAG) with VEGF (Koehler et al. 2019), here we explore the interaction of GAG with PDGF. GAG are a class of carbohydrate molecules ubiquitously present in the extracellular matrix (ECM) and at the cell membrane. These molecules act as a molecular cue in cell proliferation, regeneration and other important biological processes. GAG are linear polymers made up of repeating disaccharide units (D.U.), and they exist in different forms with variable $\mathrm{O}$ - and/or $\mathrm{N}$-sulfation patterns giving rise to different classes of GAG such as heparin (Hep), dermatan sulfate (DS), chondroitin sulfate (CS) and hyaluronic acid (HA) depending on their disaccharide composition. The relevance of GAG recognition by proteins has been well established (Samsonov and Pisabarro 2016; Vallet et al. 2020; Xu and Esko 2014). The non-immunogenic property of $\mathrm{HA}$ along with its large-scale availability by biotechnological processes make it an ideal component for innovative strategies for the development of new tissue engineering (regeneration/biomaterials) applications (Necas et al. 2008; Scharnweber et al. 2015; Xing et al. 2020). The synthesis of chemically modified HA-based oligosaccharides with different sulfation degree and pattern as well as with different functionalization such as azide and carboxytetramethylrhodamine (TAMRA) groups have been previously reported (Köhling et al. 2016; Rostovtsev et al. 2002; Tornoe et al. 2002).

In our previous studies, we have investigated the molecular recognition properties of different GAG toward various growth factors (Hintze et al. 2014; Koehler et al. 2019; Köhling et al. 2019; Rother et al. 2017, 2021; SalbachHirsch et al. 2013; Thönes et al. 2019; van der Smissen et al.
2013). Particularly, our binding studies of chemically modified tetra- and hexasaccharides (HA4 and HA6, respectively) to VEGF and HB-EGF and their delivery using collagen-based hydrogels have illustrated the relevance of such GAG derivatives for wound healing and thereof a great potential for the engineering of new biomaterials for applications in tissue regeneration (Koehler et al. 2019; Rother et al. 2017, 2021; Thönes et al. 2019). Interestingly, we have previously demonstrated that GAG act as anti-angiogenic factors in the presence of $\mathrm{VEGF}_{165}$, and, on the other hand, in the absence of $\mathrm{VEGF}_{165}$ they exhibit pro-angiogenic properties (Koehler et al. 2019), highlighting the importance of GAG as possible regulators of ECM signaling and therefore are of interest for innovative regenerative strategies.

Earlier studies on the interaction of GAG with PDGF include for instance differential binding of PDGF isoforms with GAG such as HS, DS and CS polymers carried out in mutant Chinese hamster ovary cells as well as by SPR, which suggested that polymeric GAG can bind to PDGF with high affinity (García-Olivas et al. 2003). Besides, CSA has been previously shown to enhance the mitogenic activity of PDGF in fibrosarcoma cells (Fthenou et al. 2006). Nevertheless, all so far conducted studies on the interaction of GAG with PDGF have been performed using whole cells and exclusively with GAG polymers. These studies illustrated that GAG could play an important role in regulating PDGF-mediated cellular processes; however, they did not offer molecular details on the GAG/PDGF interaction, which are crucial for understanding the regulatory mechanisms involved and to potentially exploit them to modulate the PDGF/PDGFR signaling pathway. So far, the atomic details of the interaction between GAG and PDGF and their functional mechanisms in regulation of the PDGF/PDGFR signaling system remain unknown.

Considering the dual axis control of angiogenesis by the interplay of the VEGF and PDGF systems (Bai et al. 2018; Mamer et al. 2017), and based on our previous studies on the modulation of VEGF-mediated angiogenic processes by GAG (Koehler et al. 2019), here we investigate the molecular recognition of several oligo-hyaluronan derivatives by PDGF-BB and PDGFR- $\beta$ in atomic detail taking into account the structural and functional similarity between PDGF and VEGF. Our earlier studies illustrated that defined sulfated oligo-hyaluronan derivatives can effectively bind to growth factors such as TGF- $\beta 1$, FGF-1, FGF-2 and some interleukins based on their distinct sulfation pattern, anomeric functionalization and length (Köhling et al. 2019). In particular, HA4 with different sulfation and functionalization were found to be relevant for recognition. Thus, here we decided to explore in atomic detail the interaction of PDGF-BB and PDGF- $\beta$ with four 
representative HA4 derivatives (i.e. non-sulfated HA4) anomerically functionalized with either an azide or a TAMRA functional group (HA4-N3 and HA4-TAMRA, respectively) and their per-sulfated variants (9s-HA4-N3 and 9s-HA4-TAMRA, respectively) (Figure 1A) in order to unravel the molecular mechanisms behind their recognition process and the possible functional implications. For this purpose, we have used computational methods such as docking and molecular dynamics (MD) simulations in combination with experimental binding analysis by surface plasmon resonance (SPR). Our computer-based studies predict significant differences in binding strength as well as binding site and mode based on sulfation and anomeric functionalization. Furthermore, our molecular models illustrate the functional relevance of such recognition by predicting poses in the protein-receptor recognition interface. Our computer-based predictions are in line with SPR experiments demonstrating that the studied HA4 derivatives modulate the complexation of PDGF to PDGFR- $\beta$. Our work adds insights into the molecular recognition of oligomeric HA derivatives by PDGF-BB and the implications for PDGF-BB/PDGFR- $\beta$ complex formation. This information is of value for future design of HA-based molecules with regulatory properties in terms of finetuning PDGF-mediated biological processes.

\section{Computational results}

\section{Protein and receptor structure modeling}

The complete structure of the PDGF-BB protein is not available in its unbound form in the Protein Databank (PDB). The crystal structure of the PDGF-BB dimer in complex with the receptor PDGFR- $\beta$ is available at $2.3 \AA$ resolution (PDBID 3MJG (Shim et al. 2010); Figure S2A). In this crystal structure, the PDGF-BB terminal residues 1-5 and 102-109 are missing. Those residues were modeled in extended conformation, and the whole protein structure was energy refined as described in the methods section. The resultant complete structure of PDGF-BB is shown in Figure S2 B. The terminal residues 1-5 were not organizing themselves structurally and did not appear interacting with the structured protein but remained in extended conformation, whereas residues 102-109 were organizing themselves closer to the L1 loop region of the other monomer. The resulting full structure of PDGF-BB was used as the initial structure for the GAG docking and dynamics studies (vide infra).

In the case of PDGFR- $\beta$, out of its 5 domains only 3 (D1-D3) are available in the crystal structure of the PDGF-BB/PDGFR- $\beta$ complex (PDBID 3MJG (Shim et al. 2010)) as illustrated in Figure S2 A. These three domains have been

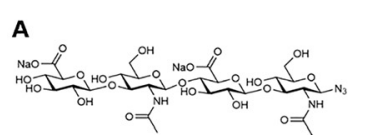

HA4-N3

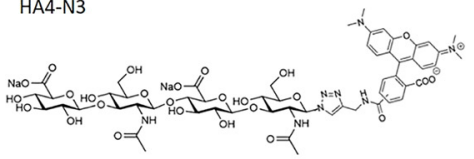

HA4-TAMRA

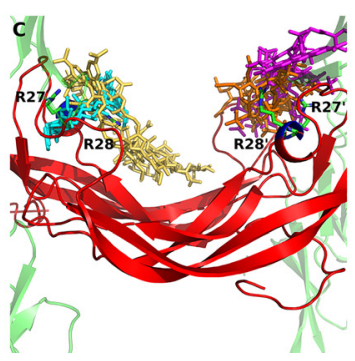

(S) $=\mathrm{SO}_{3} \mathrm{Na}$

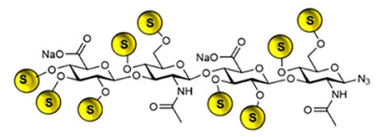

9s-HA4-N3

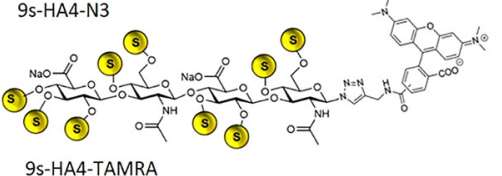

9s-HA4-TAMRA

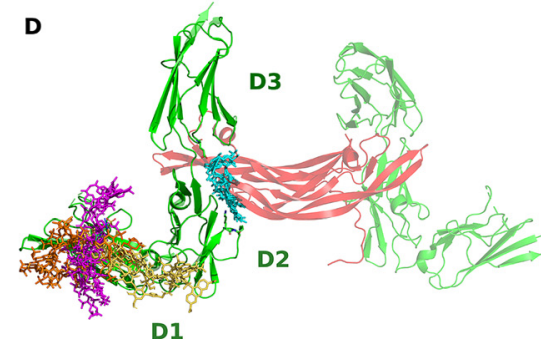

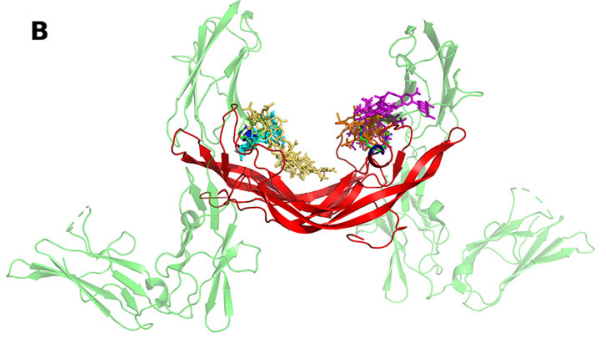

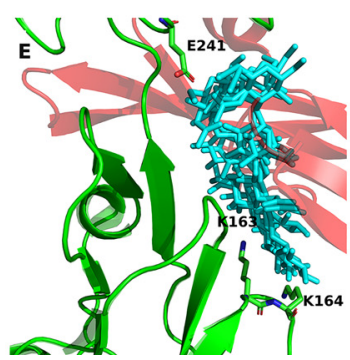

Figure 1: (A) Chemical structures of the HA4 derivatives used in our studies. (B) Clusters obtained from the docking of the HA4 variants on the PDGF-BB dimer (in red cartoon representation). The structure of PDGFR- $\beta$ is shown in green transparent cartoon for reference (i.e. it was not used in the calculations). The structures of 9s-HA4-N3, 9s-HA4-TAMRA, HA4-TAMRA and HA4-N3 are shown in orange, magenta, yellow and cyan sticks, respectively. (C) Closer view of panel (B) on the clusters obtained for the studied HA4 derivatives. The residues R27, R28 and R27', R28' (each monomer, respectively) which have been shown to be significant for recognizing PDGFR- $\beta$ are shown in sticks, labeled and colored by atom type. (D) Clusters obtained from the docking of the HA4 variants 9s-HA4-N3, 9s-HA4-TAMRA, HA4-TAMRA and HA4-N3 on PDGFR- $\beta$ (in orange, magenta, yellow and cyane sticks, respectively). The structure of PDGF-BB as well as the second receptor molecule are shown in transparent cartoon for reference (i.e. they were not used for the calculations). (E) Closer view of panel (D) on the cluster obtained for HA4-N3. The side chains of residues E241, K163 and K164 are shown in sticks, labeled and colored by atom type. 
reported to be important for interaction with PDGF-BB (Shim et al. 2010); therefore, the PDGFR- $\beta$ structure containing the D1-D3 domains was considered sufficiently relevant for the GAG docking studies. The PDGFR- $\beta$ structure is missing residues 105-110 from a loop region in the D1 domain, which we modeled as described in the methods section. The resultant complete structure of the D1-D3 domains of PDGFR- $\beta$ is shown in Figure S2 C. The modeled loop region was observed connecting the two beta-strands in the D1 domain of PDGFR- $\beta$. This structure was used as the initial structure for the GAG-docking and dynamics studies (vide infra).

\section{Analysis of the protein-receptor interface}

The protein-receptor interaction interface of the full PDGF/ PDGFR- $\beta$ complex exhibits two major interaction subinterfaces (Shim et al. 2010): a first one formed by the hydrophobic and aromatic rich cluster between PDGFR- $\beta$ D2 and PDGFR-BB, and a second one formed between the membrane-distal loops of PDGFR- $\beta$ D3 and PDGF-BB, which is predominantly hydrophobic but having also patterns of hydrogen bonding and a salt bridge at the edge (for a close view on the PDGF-BB-PDGFR- $\beta$ interface and relevant residues see Supplementary Material Figure S3). Clements and co-workers mutated amino acids throughout the PDGF-B surface and demonstrated that most mutations had only negligible effects on receptor binding, but that Arg27 and Ile30 were two major amino acids which determine PDGF-BB/PDGFR- $\beta$ interaction (Clements et al. 1991; Shim et al. 2010). The terminal guanidino group of Arg27 in PDGF-BB forms intramolecular hydrogen bonds with two main chain oxygen atoms and is buried at the protein/receptor interface forming hydrogen bonds with the main chain oxygen atom of Glu241 in PDGF- $\beta$. These intramolecular hydrogen bonds (Arg27 Ne-Phe37 O, and Arg27 Nn2-Ala35 0 ) are crucial and serve to lock the conformation of the L1 loop (residues 25-38), enabling the formation of the hydrophobic patch centered at Ala35 for receptor contact (Shim et al. 2010). The analysis of the protein-receptor interface and the available mutational studies clearly illustrate the importance of Arg27 in PDGF-B for PDGFR- $\beta$ recognition.

\section{Protein-GAG docking and representative structure selection}

The docking of the four tetrasaccharide hyaluronan-based (HA4) variants (Figure 1A) with PDGF-BB was carried out with Autodock 3 (Morris et al. 1998) as described in the methods section. The attained binding poses and modes were clustered and analyzed in order to select representative poses. The results acquired from this analysis showed HA4-N3, 9s-HA4-N3, 9s-HA4-TAMRA and HA4-TAMRA clustered around residue R27/R27' (first or second monomer, respectively) of PDGF-BB. The representative binding poses resulting from the clustering of HA4 variants with PDGF-BB are shown in Figure 1B. Interestingly, 9s-HA4-N3 and 9s-HA4-TAMRA bind to PDGF-BB in the receptor recognition region and, therefore, could potentially interfere PDGFR- $\beta$ interaction (Figure 1C). The HA4-N3 variant was found binding at other regions of PDGF-BB that are not relevant for receptor recognition, i.e. more towards the inner core region of the PDGF-BB interface (Figure 1B).

Each complex with the corresponding GAG representative structure was further energetically optimized by molecular dynamics (vide infra).

\section{Molecular dynamics of protein-GAG interaction and analysis}

The complex of PDGF-BB with each GAG representative structure resulting from the clustering analysis of the four HA4 variants was energetically refined by MD as described in methods. The binding energies are presented in Table 1.

Structural snapshots of the HA derivatives with PDGF-BB from the MD simulation are provided in Figure 2. The order of binding of HA4 derivatives to PDFG-BB was: HA4-N3 < HA4TAMRA < 9s-HA4-N3, 9s-HA4-TAMRA. As a first observation, the presence of sulfate groups and functional moieties provided a significant enhancement on the binding to PDGF-BB, being the non-sulfated HA4-N3 the variant with the weakest binding energy. During the MD simulation, HA4-N3 was observed to move towards the core cavity region of the PDGF-BB/receptor interface. Worth mentioning is the significant enhancement observed in the binding energy of nonsulfated HA4 on the addition of the functional group TAMRA. The TAMRA-substituted nona-sulfated HA4 exhibited the strongest binding energies.

The results of the per-residue interaction energy analysis are illustrated in Figure 3. From the values for the interaction of HA4-N3 and 9s-HA4-N3 with PDGF-BB, it can be appreciated that the presence of sulfate groups in 9s-HA4-N3 significantly enhances its binding to PDGF-BB in comparison to the non-sulfated HA4-N3 (Figure 3A; azide group and residues 1-4 for sugar moieties). The values obtained for HA4-TAMRA and 9s-HA4-TAMRA reflect the significant effect of the functional group TAMRA in increasing the binding to PDGF-BB (Figure 3B).

The favorable interaction observed represents the rhodamine group of this functionalization. Closer analysis 
Table 1: MM-GBSA binding free energy values of the studied HA4 derivatives with PDGF-BB and PDGFR- $\beta$ ( $\mathrm{kcal} / \mathrm{mol}$ ).

\begin{tabular}{lrrrrr}
\hline GAG variant & \multicolumn{2}{c}{ PDGF-BB } & \multicolumn{2}{c}{ PDGFR- $\beta$} \\
\cline { 2 - 6 } & Clus1 & Clus2 & & Clus1 & Clus2 \\
\hline HA4-N3 & $-9.1 \pm 4.5$ & - & $4.3 \pm 0.6$ & $-10.6 \pm 5.1$ & $3.8 \pm 0.5$ \\
9S-HA4-N3 & $-68.2 \pm 13.9$ & - & $-0.2 \pm 4.2$ & - & - \\
HA4-TAMRA & $-32.0 \pm 4.6$ & - & $-13.0 \pm 2.8$ & - \\
9s-HA4-TAMRA & $-57.5 \pm 5.6$ & $-70.1 \pm 6.5$ & $-4.8 \pm 6.0$ & $18.8 \pm 3.9$ & - \\
\hline
\end{tabular}

A
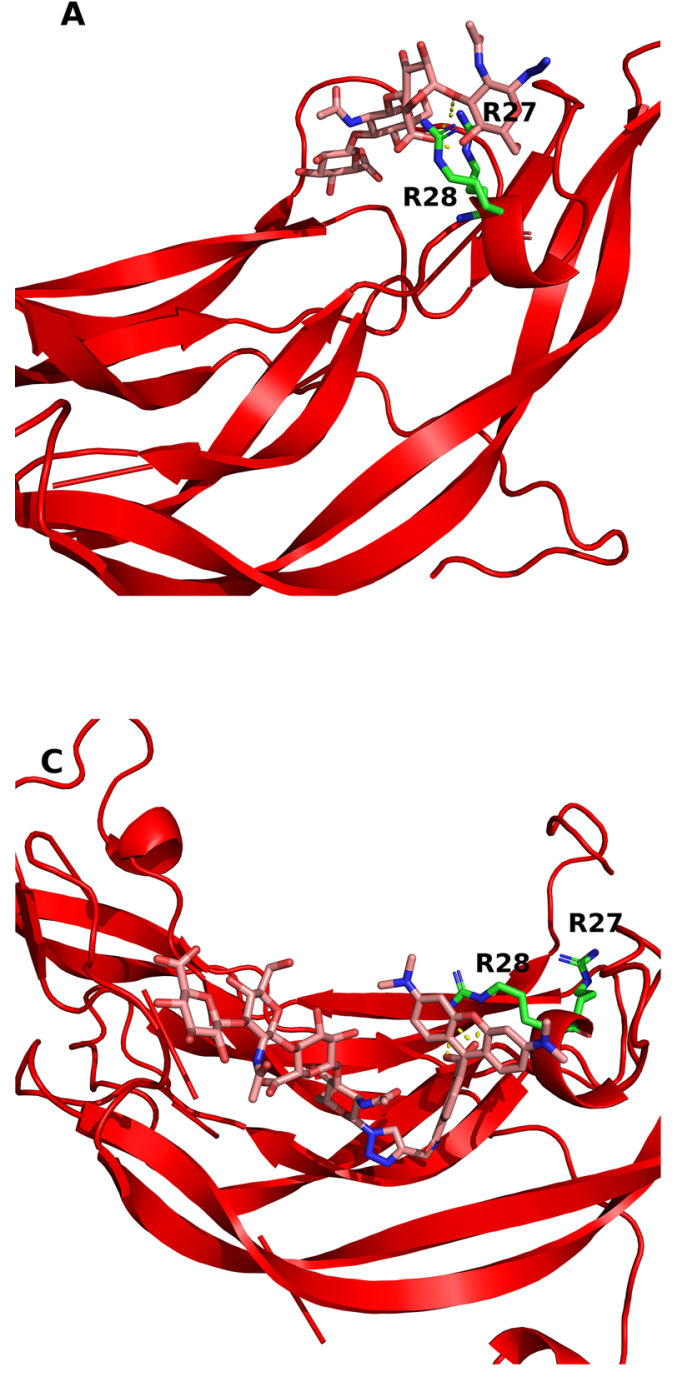

B

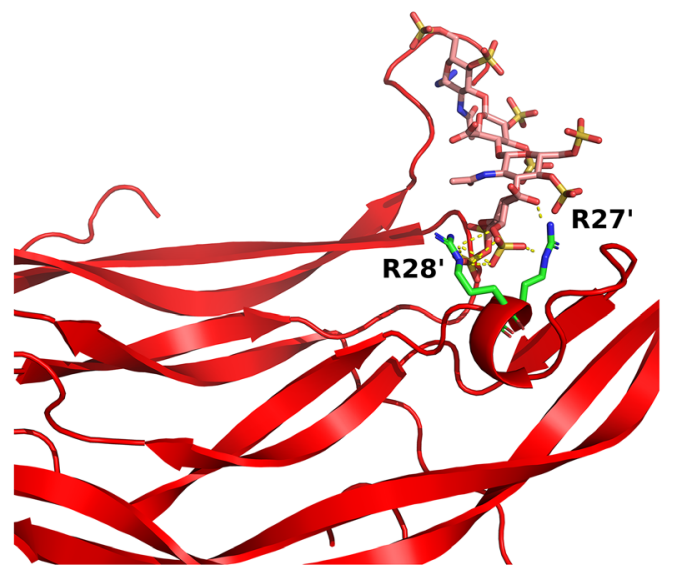

D

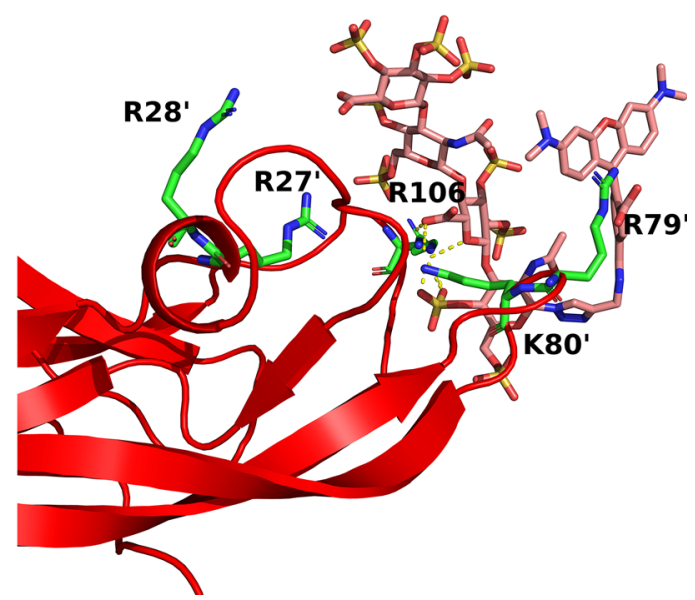

Figure 2: Snapshot of the MD simulation of PDGF-BB in complex with the representative structures of the docked HA4 derivatives (A) HA4-N3, (B) 9s-HA4-N3, (C) HA4-TAMRA and (D) 9s-HA4-TAMRA.

PDGF-BB is shown in red cartoon. The side chain of relevant protein residues and the HA4 derivatives are shown in atom-colored sticks (green and pink for carbons, respectively).

of the MD results illustrates the cation- $\pi$ interaction between the rhodamine group of TAMRA and Arg side chains in PDGF-BB (Figure S4). It is worth noting that with the inclusion of sulfate groups into HA4 the contribution of the
TAMRA decreases, which in turn is compensated by the gain in binding energy of the sulfate groups in the sugar component (residues 1-4) of the HA4 molecule. Thus, there is a distinguishable effect on growth factor recognition 

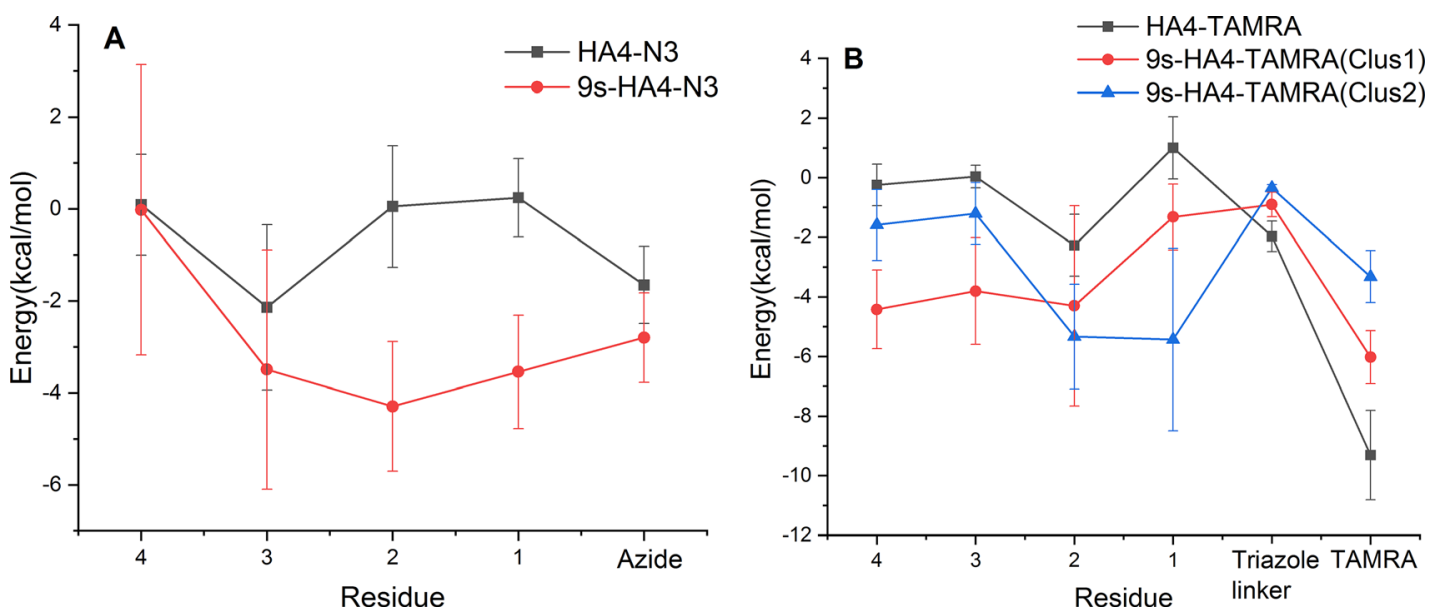

Figure 3: Per-residue interaction energy of the studied HA4 derivatives with PDGF-BB.

(A) N3 derivatives. (B) TAMRA derivatives. Residue 1-4 refers to 1-4 sugar moieties. The errors bars represent the standard deviation.

depending on the sulfation and functionalization, which can be rationalized based on our molecular models.

The significant per-residue binding energies of PDGF-BB residues interacting with HA4 derivatives were also analyzed (Table 2). The results indicated significant binding energy of derivatives 9s-HA4-N3, 9s-HA4-TAMRA and HA4-TAMRA to residues R27 and R27' in each monomer of PDGF-BB, respectively, and the neighboring region, which might be relevant for PDGFR- $\beta$ recognition. In the case of 9s-HA4-TAMRA, although the interaction observed with R27' is weak, we could observe that this GAG derivative binds strongly to the loop regions which act as entry point to access to R27', thus being able to disrupt PDGF-BB/ PDGFR- $\beta$ recognition (Figure 2D). HA4-N3 was observed interacting with other regions of PDGF-BB which are not involved in receptor recognition as evident by the obtained per-residue binding energy values.

\section{Molecular dynamics of receptor-GAG interaction and analysis}

The complex of PDGFR- $\beta$ with each GAG representative structure selected from clustering analysis of the four HA4 variants was energetically refined by MD as described in methods. The obtained results are presented in Table 1 . The binding energy analysis showed very weak binding of HA4-N3 (Clus2) to the D1-D3 domain regions of PDGFR- $\beta$ (in the range of $\sim-10 \mathrm{kcal} / \mathrm{mol}$ ). Furthermore, for HA4-N3 (Clus1 and 3), HA4-TAMRA (Clus2) and 9s-HA4-TAMRA (Clus2) positive binding energy values were observed, clearly indicating no interaction with the receptor.

The per-residue binding energy values for the interaction of HA4-N3 with PDGFR- $\beta$ are shown in Table 2, in which it can be observed the predicted weak binding to

Table 2: Per-residue binding free energy for the studied HA4 derivatives with PDGF-BB and PDGFR- $\beta$ ( $\mathrm{kcal} / \mathrm{mol})$. Residues known to be relevant for molecular recognition are highlighted in bold.

\begin{tabular}{|c|c|c|c|c|}
\hline \multirow[t]{2}{*}{ GAG variant } & \multicolumn{2}{|c|}{ PDGF-BB } & \multicolumn{2}{|c|}{ PDGFR- $\beta$} \\
\hline & Clus1 & Clus2 & Clus1 & Clus2 \\
\hline HA4-N3 & - & - & - & $\begin{array}{l}\mathrm{K} 163(-2.4 \pm 1.4) \\
\mathrm{K} 164(-2.1 \pm 1.3) \\
\mathrm{Y} 207(-3.5 \pm 0.8)\end{array}$ \\
\hline 9s-HA4-N3 & $\begin{array}{l}\text { R56 }(-11.6 \pm 2.2) R 106(-5.1 \pm 1.9) \\
R 27^{\prime}(-14.5 \pm 4.8) R 28^{\prime}(-6.7 \pm 4.2) \\
R 32^{\prime}(-4.2 \pm 3.5)\end{array}$ & - & & \\
\hline HA4-TAMRA & $\begin{array}{l}\mathbf{R} 28(-8.8 \pm 1.0) \mathrm{L} 29(-2.5 \pm 0.4) \\
\operatorname{R} 61(-4.9 \pm 1.2)\end{array}$ & - & & \\
\hline 9s-HA4-TAMRA & $\begin{array}{l}\text { R32(-9.2 } \pm 1.5) \text { T33 }(-2.8 \pm 0.5) \\
\text { N34 }(-1.5 \pm 0.5) \mathrm{K} 85(-5.1 \pm 1.5) \\
\text { K86(-7.5 } \pm 1.9) \text { S1'(3.9 } \pm 2.2)\end{array}$ & $\begin{array}{l}R 56(-3.3 \pm 1.8) \text { A104 }(-2.1 \pm 0.5) \\
A 105(-2.2 \pm 0.8) R 106(-16.9 \pm 1.9) \\
\text { V108(-3.1 } \pm 1.1) R 27^{\prime}(-2.4 \pm \mathbf{2 . 3}) \\
R 79^{\prime}(-7.7 \pm 0.9) \mathrm{K} 80^{\prime}(-8.7 \pm 2.0) \\
K 81^{\prime}(-2.2 \pm 0.8)\end{array}$ & & \\
\hline
\end{tabular}


residues $\mathrm{K} 163$ and $\mathrm{K} 164$, which are located in a relevant region for the recognition of PDGF-BB (Shim et al. 2010).

\section{Experimental results}

\section{Binding of HA4 derivatives to immobilized PDGF-BB}

The interaction of solute HA4 derivatives with immobilized PDGF-BB was analyzed by SPR as described in the methods section. The ranking of binding strength of the HA4 derivatives with PDGF-BB was as follows: HA4-N3 < HA4TAMRA 9s-HA4-N3 < 9s-HA4-TAMRA (Figure 4A). The results obtained clearly demonstrate the impact of GAG modification with TAMRA, as those derivatives (HA4-TAMRA and 9s-HA4-TAMRA) showed a higher binding strength compared to their counterparts lacking this modification. These experimental observations are in line with the computational predictions in terms of a clear enhancement of GAG binding energy with increase in sulfate content as well as with the presence of TAMRA.

\section{Binding of PDGF-BB to immobilized PDGFR- $\beta$ in the presence of $\mathrm{HA} 4$ derivatives}

HA4 derivatives incubated with PDGF-BB affected receptor recognition in a sulfation- and concentration-dependent manner (Figure 4B), while HA4 derivatives when added alone to immobilized PDGFR- $\beta$ showed weak interaction, as predicted by our theoretical models. Pre-incubation of PDGF-BB with the derivatives HA4-TAMRA, 9s-HA4-N3 and 9s-HA4-TAMRA significantly reduced the complexation of growth factor and receptor, while in the presence of HA4-N3 no inhibitory effect was observed. Among the HA4 derivatives, 9s-HA4-TAMRA revealed the strongest blocking potential for protein-receptor recognition. Thus, the complexation of PDGF-BB and PDGFR- $\beta$ is hindered by the presence of HA4 derivatives in the order of HA4TAMRA 9s-HA4-N3 < 9s-HA4-TAMRA. Complete blocking of growth factor-receptor complexation was, however, not observed at the GAG concentrations investigated.

From the computational and experimental results, it can be concluded that the studied HA4 derivatives can bind to PDGF-BB in a sulfation- and functionalization(TAMRA group) dependent manner, and that such HA4 derivatives have a very weak interaction with PDGFR- $\beta$. The 9s-HA4-TAMRA derivative was found to interfere with the PDGF-BB-PDGFR- $\beta$ interaction at micromolar concentration $(15-150 \mu \mathrm{M})$.

These results illustrate the potential of 9s-HA4 derivatives with particular functionalization such as TAMRA for the design of molecules with inhibitory properties of PDGF-BB/PDGFR- $\beta$ signaling.

\section{Binding of HA4 derivatives to immobilized PDGFR- $\beta$}

The derivative 9s-HA4-TAMRA showed a direct and concentration-dependent binding to PDGFR- $\beta$ in contrast
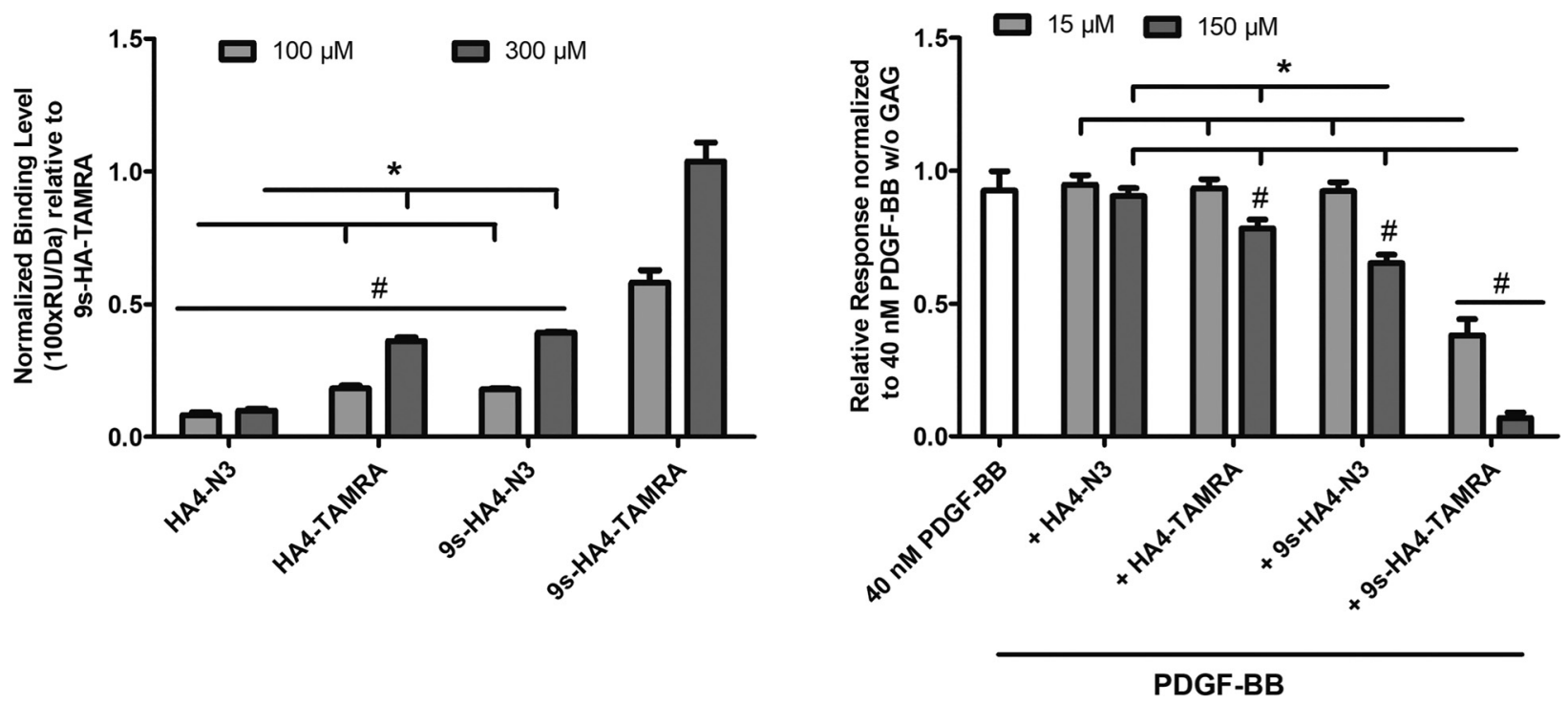

Figure 4: (A) Binding levels for the interaction of immobilized PDGF-BB with solute HA4 derivatives as determined by SPR. All values represent the mean $\pm \mathrm{SD}$ of $n=3$ and are given as relative to baseline response and corrected for the respective molecular weight of HA4 derivatives. Twoway ANOVA: ${ }^{*} p<0.05$ versus respective treatment; $\# p<0.05$ versus $9 \mathrm{~s}-$ HA4-TAMRA. (B) Binding levels for the interaction of immobilized PDGFR- $\beta$ with $40 \mathrm{nM}$ PDGF-BB alone or after pre-incubation with 15 and $150 \mu$ M HA4 derivatives. All values represent the mean \pm SD of $n=3$ and are given as relative to baseline response. One-way ANOVA: ${ }^{\star} p<0.05$ versus respective treatment; $\# p<0.05$ versus 40 nM PDGF-BB. 

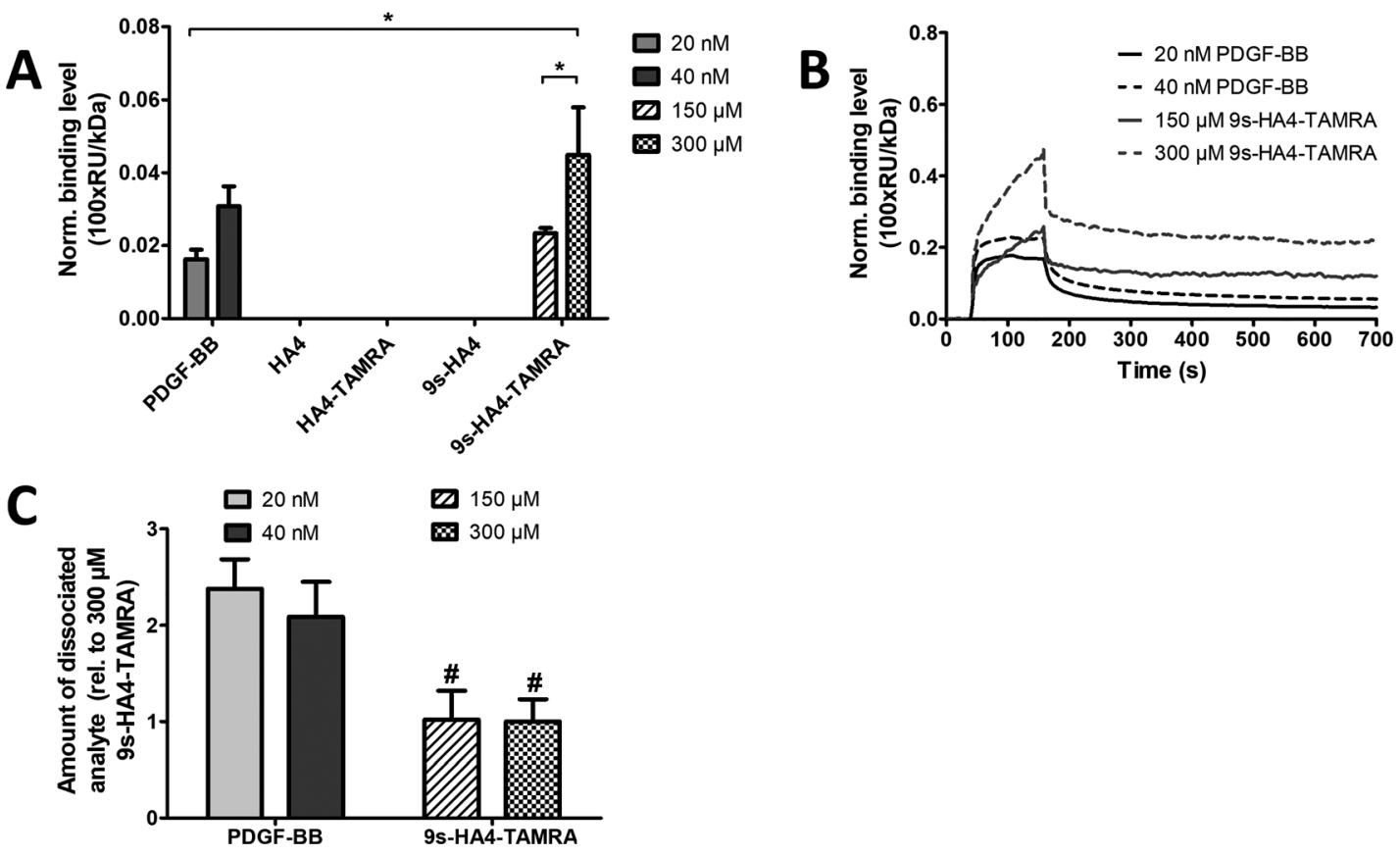

Figure 5: Binding of HA4 derivatives to immobilized PDGFR- $\beta$ in comparison to PDGF-BB analyzed via SPR.

(A) Binding levels of the interaction were recorded $10 \mathrm{~s}$ before injection stop and corrected for the respective molecular weight of the analyte. (B) Sensorgram of the interaction of 150 and $300 \mu \mathrm{M} 9 \mathrm{~s}-\mathrm{HA} 4-$ TAMRA with the receptor compared to 20 and $40 \mathrm{nM}$ PDGF-BB. Binding response was corrected for the respective molecular weight of the analytes. (C) The relative amount of dissociated analyte from PDGFR- $\beta$ was calculated from the slope of the dissociation phase. For (A) and (C) values represent the mean $\pm S D$ for $n=3$. For (B) one representative curve from three measurements is shown. One-way ANOVA: ${ }^{*} p<0.05$ versus Respective treatment, $\# p<0.05$ versus 20 and 40 nM PDGF-BB.

to all other HA4 derivatives, which did not exhibit binding to the receptor. Nevertheless, the needed concentrations for 9s-HA4-TAMRA to obtain binding (150 and $300 \mu \mathrm{M}$, respectively) were higher compared to the ones for PDGF-BB (20 and $40 \mathrm{nM}$ ) (Figure 5A). Binding curves for 9s-HA4-TAMRA and PDGF-BB appeared to be different, as for 9s-HA4-TAMRA no equilibrium between association and dissociation of the analyte during its injection was achieved in contrast to PDGF-BB (Figure 5B). Furthermore, less 9s-HA4-TAMRA dissociated from the receptor during dissociation phase compared to PDGF-BB, which indicates a strong interaction of the GAG derivative with PDGFR- $\beta$ at the tested concentrations (Figure $5 \mathrm{C}$ ). However, with regard to the higher concentrations of 9s-HA4-TAMRA that were used ( $\mu \mathrm{M}$ vs. $\mathrm{nM})$, its binding strength appears to be weaker compared to PDGF-BB. It is worth mentioning that our theoretical models also pointed out the weak binding of HA derivatives to PDGFR- $\beta$. Whereas our models only included domains D1-D3 of PDGF-BB which are relevant for receptor recognition, the experimental studies included domains D1-D5 of PDGFR- $\beta$. Therefore, some binding might occur in regions of D4-D5 which are not relevant for PDGF-BB/receptor recognition.

\section{Discussion}

Growth factors such as VEGF and PDGF are demonstrated to play a major role in angiogenesis (Bai et al. 2018; Mamer et al. 2017), which is a very important process in wound healing due to the fact that most tissue types like skin and bone are vascularized. The role of VEGF in angiogenesis is well studied and established. The effect of GAG as proangiogenic or anti-angiogenic agents in VEGF/VEGFR mediated angiogenesis has been recently established (Koehler et al. 2019). In the case of PDGF-mediated angiogenesis, the so-far available studies on the effect of GAG are limited and are mostly with polymeric GAG and lacking structural and mechanistic details (García-Olivas et al. 2003; Fthenou et al. 2006). Most importantly, the available studies on PDGF/GAG interactions have been carried out with naturally occurring GAG such as polymeric HS, DS and CS, 
which indeed shows a vital role of GAG in modulating the proliferation process. Chemically defined HA4 derivatives with different degrees of sulfation have been shown to be effective in binding and modulating the bioactivity of various growth factors which in turn influence healing and regeneration process (Koehler et al. 2019; Rother et al. 2017, 2021; Thönes et al. 2019). Based on our previous knowledge of GAG-mediated angiogenesis modulation of the VEGF/ VEGFR system, here we attempted to explore the interaction of GAG with the analogous PDGF-BB/PDGFR- $\beta$ system, which is also an interesting target to tackle for angiogenesis regulation. The aim of this study has been to understand the mode of interaction of a set of representative oligomeric HA4 derivatives of different sulfation and functionalization with the PDGF-BB/PDGFR- $\beta$ system and the potential functional implications. For this purpose, and in order to gain atomic and mechanistic details, here we apply molecular docking and MD simulation techniques to obtain theoretical models that allow us to gain new structure-based insights into the molecular recognition process and its functional implications. Furthermore, our computer based work is combined with experimental SPR binding studies.

The results from the computational investigation show that the studied oligomeric HA4 derivatives interact with PDGF-BB and PDGFR- $\beta$ in a different manner; indeed, with strong binding affinity to the PDGF-BB while insignificant to PDGFR- $\beta$. The binding to PDGF-BB is affected by sulfation, which clearly plays a major role in dictating the binding affinity of the studied HA4 derivatives to PDGF-BB and is illustrated by the higher binding energy of 9s-HA4 derivatives versus the non-sulfated variants. The functionalization of the HA4 derivative also influences its recognition to PDGF-BB, which is exemplified by the higher binding strength of the TAMRA derivatives with respect to the azido counterparts. The experimental SPR results fully agree with the computational predictions as they show that the sulfation and functionalization with TAMRA attributes a great impact on the binding strength of the studied HA4 derivatives to PDGF-BB.

In terms of the functional implications derived from our studies, the distinguishable site and mode of interaction obtained for each of the studied HA4 derivatives is of great interest and it is also supported by the SPR experiments. HA4-TAMRA, 9s-HA4-TAMRA and 9s-HA4-N3 are predicted to be interacting with PDGF-BB at sites which are relevant for receptor recognition. The complexation of PDGF-BB and PDGFR- $\beta$ is demonstrated by SPR to be precluded by the per-sulfated and TAMRA HA4 derivatives.

The observed high binding strength makes hyaluronanbased tetrasaccharide derivatives promising candidates for being exploited to modulate the PDGF-BB/PDGFR complexation process and potentially the signaling of this macromolecular system. For such potential future biological applications, the interaction of such GAG derivatives with other proteins involved in the biological process should be investigated in detail. VEGF and PDGF function in a tandem fashion in the process of angiogenesis, whereas GAG have been earlier demonstrated to be able to modulate VEGF-mediated angiogenesis. Thus, this study opens up the possibility that this kind of GAG may be used as a single molecular entity with the potential to effectively modulate VEGF- and PDGF-mediated signaling. Our molecular models will be of value to guide future investigations along these lines.

\section{Materials and methods}

\section{Molecular modeling of HA4 derivatives}

The modeling of the structures of tetrasaccharide HA4 and 9s-HA4 (as Azide and TAMRA derivatives) has been previously reported (Köhling et al. 2019). The structure of the GAG molecules used in this study are shown in Figure 1A.

\section{Molecular modeling of protein and receptor}

The structure of the PDGF-BB/PDGFR- $\beta$ complex was obtained from the Protein Data Bank (Berman et al. 2000) (PDBID 3MJG, 2.3Å resolution (Shim et al. 2010)). The missing terminal residues $1-5$ and 102109 of PDGF-BB were modeled in extended conformation using PyMOL (Schrödinger 2009-2015). The residues 105-110 from a loop region in the D1 domain, which were missing in the PDGFR- $\beta$ receptor structure were modeled using the Swiss-Model server (Waterhouse et al. 2018).

The full structure of PDGF-BB (residues 1-109) and the D1-D3 domains of PDGFR- $\beta$ (residues 33-314) were energetically refined separately by MD simulation using AMBER 14 (Case et al. 2014). The ff14SB force field parameters were used. Each system, protein and receptor separately, was solvated in a truncated octahedral box of TIP3P water molecules and neutralized with $\mathrm{Na}^{+}$or $\mathrm{Cl}^{-}$counterion. The energy refinement protocol used was as follows: PDGF-BB was treated fully flexible, whereas for PDGFR- $\beta$ only the modeled loop region was left unrestrained. The rest of the receptor structure was fixed with weak positional restraints $(10 \mathrm{kcal} /$ $\mathrm{mol} / \AA^{2}$ ) during the MD simulation. The protein structure as well as the receptor were energetically refined individually by $25 \mathrm{~ns}$ of MD simulation each. The resulting energy optimized structures of PDGF-BB and PDGFR- $\beta$ were used for the docking calculations.

\section{Molecular docking of GAG to protein and receptor}

Docking was carried out with Autodock 3 (Morris et al. 1998) to predict the binding of derivatives HA4-N3, 9s-HA4-N3, HA4-TAMRA and 9sHA4-TAMRA to PDGF-BB and to PDGFR- $\beta$. Autogrid3 was used to calculate the atomic potential of each full structure with a grid box and spacing grid of $124 \times 126 \times 126 \AA$ and $0.602 \AA$ for PDGF-BB and of 
$126 \times 126 \times 126 \AA$ and $0.736 \AA$ PDGFR- $\beta$. HA4 derivatives were treated flexible, whereas protein and receptor were maintained rigid. A total of 1000 independent runs were carried out with the Lamarckian genetic algorithm (initial population size: 300, termination condition: 10,000 generations, energy evaluations: $9995 \times 10^{5}$ ). The top 50 docking solutions in each case (i.e. protein and receptor) were clustered with DBSCAN (Ester et al. 1996) as previously described (Gehrcke and Pisabarro 2015). For PDGF-BB and PDGFR- $\beta$, a representative pose from each HA4 derivative cluster was selected for further energy refinement.

\section{Molecular dynamics (MD) simulations}

Each GAG/PDGF-BB and GAG/PDGFR- $\beta$ complex selected as representative from the docking studies was further energetically optimized by molecular dynamics (MD) simulations using AMBER14 (Case et al. 2014) as previously described (Köhling et al. 2019; Koehler et al. 2019). Charges were taken from the GLYCAM 06-j force field (Kirschner et al. 2008) for the HA units and from the literature for sulfate groups (Huige and Altona 1995). Parameters for the HA part were taken from the GLYCAM-06j force field and for the proteins from the ff14SB force field (Case et al. 2014). Libraries and parameters for the functionalized derivatives were provided from Köhling et al. (2019). Each GAG/PDGF-BB and GAG/PDGFR- $\beta$ complex was solvated in a truncated octahedral box of TIP3P water molecules and neutralized with $\mathrm{Na}^{+}$or $\mathrm{Cl}^{-}$counterions. MD simulations were preceded by two energy-minimization steps: (i) only the solvent and ions were relaxed with position restraints for the solute $\left(500 \mathrm{kcal} / \mathrm{mol} / \AA^{2}\right)$ using 1000 steps of steepest descendent minimization followed by 500 steps of conjugate gradient minimization; (ii) the entire system was energy-minimized without restraints applying 3000 cycles of steepest descendent and 3000 steps of conjugate gradient equilibration. Then, the system was heated up from 200 to $300 \mathrm{~K}$ in 20 ps with weak position restraints $(10 \mathrm{kcal} /$ mol. $\AA^{2}$ ). The pyranose rings in the HA molecules were harmonically restrained. Langevin temperature coupling with a collision frequency $y=1 \mathrm{ps}^{-1}$ was used at this step. The whole system was equilibrated under constant pressure of $1 \mathrm{~atm}$ using periodic boundary conditions (NPT conditions) at $300 \mathrm{~K}$ for $50 \mathrm{ps}$. A total of $30 \mathrm{~ns} \mathrm{MD}$ simulation was carried out at $300 \mathrm{~K}$ NPT conditions for each complex. All bonds involving hydrogen atoms were constrained using SHAKE algorithm. A time step of 2 fs was used. A cutoff of $8 \AA$ was applied to treat the non-bonded interactions, and the Particle Mesh Ewald (PME) method was used to treat long-range electrostatic interactions. MD trajectories were recorded every 10 ps. Trajectories were visualized with VMD (Humphrey et al. 1996). Energy decomposition per residue as well as binding free energy postprocessing analysis of the last 200 frames from the MD simulations were performed in implicit solvent using the MM-GBSA method (Miller et al. 2012; Wang et al. 2001) as implemented in AMBER14. Data analysis was carried out with OriginLab (OriginLab 2019). Figures were created with PyMOL (Schrödinger 2009-2015).

\section{Experimental methods}

\section{Synthesis of HA4 derivatives}

The HA4 derivatives were synthesized using the protocols published earlier in Köhling et al. (2016, 2019).

\section{Proteins}

Recombinant human PDGF-BB (220-BB) and PDGFR- $\beta$ (358-PR/CF) were purchased from R\&D Systems (Wiesbaden-Nordenstadt, Germany).

\section{Immobilization of PDGF-BB and PDGFR- $\beta$ on SPR sensor chips}

SPR analysis was performed with a BIACORE T200 instrument (GE Healthcare). PDGF-BB and PDGFR- $\beta$ were immobilized on the surface of Series S Sensor Chip CM5 at $25^{\circ} \mathrm{C}$ in HBS-EP running buffer $(0.01 \mathrm{M}$ HEPES (pH 7.4), $0.15 \mathrm{M} \mathrm{NaCl}, 3 \mathrm{mM}$ EDTA, 0.055\% surfactant P20) using the amine coupling reaction according to the manufacturer's protocol. $10 \mu \mathrm{g} / \mathrm{ml}$ protein diluted in sodium acetate buffer ( $\mathrm{pH} 5.5$ for PDGF-BB and $\mathrm{pH} 5.0$ for PDGFR- $\beta$ ) were injected at $5 \mu \mathrm{L} / \mathrm{min}$ until an immobilization level of 3000 RU for PDGF-BB and 1300 RU for PDGFR- $\beta$ was achieved. As a reference one flow cell was activated and directly deactivated without immobilizing the protein.

\section{SPR analysis of GAG binding to immobilized PDGF-BB}

Interaction study was performed as described previously (Koehler et al. 2019) Briefly, HA4 derivatives (diluted in HBS-EP) were injected for $300 \mathrm{~s}$ at $30 \mu \mathrm{L} / \mathrm{min}$ (at $37^{\circ} \mathrm{C}$ in HBS-EP) and binding levels were recorded $10 \mathrm{~s}$ before injection stop. The injection was followed by a $10 \mathrm{~min}$ dissociation phase in running buffer at a flow rate of $30 \mu \mathrm{L} / \mathrm{min}$. After each sample injection the sensor chip surface was regenerated with two pulses (60 s) of $5 \mathrm{M} \mathrm{NaCl}$ in $35 \mathrm{mM} \mathrm{NaOH}$ at $30 \mu \mathrm{L} / \mathrm{min}$. The baseline was allowed to stabilize for $1000 \mathrm{~s}$ with running buffer prior to injection of the next sample. BIACORE evaluation software 2.03. was used to evaluate binding parameters. Data were double referenced by the response of the reference surface and the response of HBS-EP buffer alone relative to a baseline report point. To consider the fact that the SPR binding response is related to a mass increase at the sensor chip surface, binding levels were corrected for the respective molecular weight of the HA4 derivatives.

\section{SPR analysis of PDGF-BB binding to PDGFR- $\beta$ in the presence of HA4 derivatives}

The chip surface with immobilized PDGFR- $\beta$ was blocked with $1 \%$ $\mathrm{BSA}, 5 \%$ sucrose in HBS-EP $(3 \times 700 \mathrm{~s}$ at $30 \mu \mathrm{L} / \mathrm{min})$ prior to interaction analysis. 15 and $150 \mu \mathrm{M}$ of the respective HA4 derivatives were preincubated for $60 \mathrm{~min}$ at RT with $40 \mathrm{nM}$ PDGF-BB in HBS-EP. After three start up injections, PDGF-BB with and without HA4 derivatives was injected for $120 \mathrm{~s}$ at $30 \mu \mathrm{L} / \mathrm{min}$. The injection was followed by a dissociation phase of $600 \mathrm{~s}$ in running buffer at $30 \mu \mathrm{L} / \mathrm{min}$. The sensor surface was regenerated after each sample injection with an injection of $10 \mathrm{mM}$ glycine- $\mathrm{HCl} \mathrm{pH} 2.0$ for $30 \mathrm{~s}(30 \mu \mathrm{L} / \mathrm{min})$ followed by $5 \mathrm{M}$ sodium chloride in $35 \mathrm{mM} \mathrm{NaOH}$ for $60 \mathrm{~s}(30 \mu \mathrm{L} / \mathrm{min})$. The baseline was allowed to stabilize for $1000 \mathrm{~s}$ with running buffer prior to injection of the next sample. Data were double referenced by the response of the reference surface and the preceding response of HBS-EP buffer relative to a baseline report point. 


\section{SPR analysis of HA derivatives binding to PDGFR- $\beta$}

150 and $300 \mu \mathrm{M}$ of HA4 derivative diluted in HBS-EP were injected over immobilized PDGFR- $\beta$ for $120 \mathrm{~s}$ at $30 \mu \mathrm{L} / \mathrm{min}$. As a control, 20 and $40 \mathrm{nM}$ PDGF were injected as well. The injections were followed by a dissociation phase of $10 \mathrm{~min}$ in running buffer at $30 \mu \mathrm{L} / \mathrm{min}$. Regeneration was performed with $10 \mathrm{mM}$ Glycine- $\mathrm{HCl}(\mathrm{pH} 2.0)$ for $30 \mathrm{~s}$ at $30 \mu \mathrm{L} / \mathrm{ml}$ followed by $5 \mathrm{M} \mathrm{NaCl}$ in $35 \mathrm{mM} \mathrm{NaOH}$ for $60 \mathrm{~s}$ at $30 \mu \mathrm{L} / \mathrm{min}$. Data were double referenced and corrected for the respective molecular weight of the analytes.

Acknowledgments: We acknowledge financial support by the German Research Council (DFG TransRegio67, subprojects A3, A7, A8, B1 and Z3). We are thankful to Pedro Manuel Guillem-Gloria for his extraordinary computational technical support, and to Dr. Gloria Ruiz-Gómez for providing libraries and parameters for the functionalized HA derivatives.

Author contributions: All the authors have accepted responsibility for the entire content of this submitted manuscript and approved submission.

Research funding: German Research Council (DFG TransRegio67, project number 59307082, subprojects A3, A7, A8, B1 and Z3.

Conflict of interest statement: The authors declare no conflicts of interest regarding this article.

\section{References}

Andrae, J., Gallini, R., and Betsholtz, C. (2008). Role of plateletderived growth factors in physiology and medicine. Genes Dev. 22: 1276-1312.

Bai, Y., Bai, L., Zhou, J., Chen, H., and Zhang, L. (2018). Sequential delivery of VEGF, FGF-2 and PDGF from the polymeric system enhance HUVECS angiogenesis in vitro and CAM angiogenesis. Cell. Immunol. 323: 19-32.

Banfi, A., von Degenfeld, G., Gianni-Barrera, R., Reginato, S., Merchant, M.J., McDonald, D.M., and Blau, H.M. (2012). Therapeutic angiogenesis due to balanced single-vector delivery of VEGF and PDGF-BB. Faseb. J. 26: 2486-2497.

Berman, H.M., Westbrook, J., Feng, Z., Gilliland, G., Bhat, T.N., Weissig, H., Shindyalov, I.N., and Bourne, P.E. (2000). The protein data bank. Nucleic Acids Res. 28: 235-242.

Case, D.A., Berryman, J.T., Betz, R.M., Cerutti, I.D.S., Cheatham, T.E., Darden, T.A., Duke, R.E., Giese, T.J., Gohlke, H., Goetz, A.W., et al. (2014). Amber 2014. San Francisco: University of California, https://ambermd.org/.

Clements, J.M., Bawden, L.J., Bloxidge, R.E., Catlin, G., Cook, A.L., Craig, S., Drummond, A.H., Edwards, R.M., Fallon, A., and Green, D.R. (1991). Two PDGF-B chain residues, arginine 27 and isoleucine 30, mediate receptor binding and activation. EMBO J. 10: 4113-4120.

Erber, R., Thurnher, A., Katsen, A.D., Groth, G., Kerger, H., Hammes, H., Menger, M.D., Ullrich, A., and Vajkoczy, P. (2004). Combined inhibition of VEGF and PDGF signaling enforces tumor vessel regression by interfering with pericyte-mediated endothelial cell survival mechanisms. Faseb J. 18: 338-340.
Ester, M., Kriegel, H.-P., Sanders, J., and Xu, X. (1996). A densitybased algorithm for discovering clusters in large spatial databases with noise. In: Proceedings of 2 nd international knowledge discovery and data mining. (KKD96), pp. 226-231.

Fredriksson, L., Li, H., and Eriksson, U. (2004). The PDGF family: four gene products form five dimeric isoforms. Cytokine Growth Factor Rev. 15: 197-204.

Fthenou, E., Zafiropoulos, A., Tsatsakis, A., Stathopoulos, A., Karamanos, N.K., and Tzanakakis, G.N. (2006). Chondroitin sulfate $A$ chains enhance platelet derived growth factormediated signalling in fibrosarcoma cells. Int. J. Biochem. Cell Biol. 38: 2141-2150.

García-Olivas, R., Hoebeke, J., Castel, S., Reina, M., Fager, G., Lustig, F., and Vilaró, S. (2003). Differential binding of platelet-derived growth factor isoforms to glycosaminoglycans. Histochem. Cell Biol. 120: 371-382.

Gehrcke, J.P. and Pisabarro, M.T. (2015). Identification and characterization of a glycosaminoglycan binding site on interleukin-10 via molecular simulation methods. J. Mol. Graph. Model. 62: 97-104.

Gialeli, C., Nikitovic, D., Kletsas, D., Theocharis, A.D., Tzanakakis, G.N., and Karamanos, N.K. (2014). PDGF/PDGFR signaling and targeting in cancer growth and progression: focus on tumor microenvironment and cancer-associated fibroblasts. Curr. Pharmaceut. Des. 20: 2843-2848.

Gianni-Barrera, R., Butschkau, A., Uccelli, A., Certelli, A., Valente, P., Bartolomeo, M., Groppa, E., Burger, M.G., Hlushchuk, R., Heberer, M., et al. (2018). PDGF-BB regulates splitting angiogenesis in skeletal muscle by limiting VEGF-induced endothelial proliferation. Angiogenesis 21: 883-900.

Hintze, V., Samsonov, S.A., Anselmi, M., Moeller, S., Becher, J., Schnabelrauch, M., Scharnweber, D., and Pisabarro, M.T. (2014). Sulfated glycosaminoglycans exploit the conformational plasticity of bone morphogenetic protein-2 (BMP-2) and alter the interaction profile with its receptor. Biomacromolecules 15: 3083-3092.

Huige, C.J.M. and Altona, C. (1995). Force-field parameters for sulfates and sulfamates based on ab-initio calculations - extensions of Amber and Charmm fields. J. Comput. Chem. 16: 56-79.

Humphrey, W., Dalke, A., and Schulten, K. (1996). VMD: Visual molecular dynamics. J. Mol. Graph. Model. 14: 33-38.

Kirschner, K.N., Yongye, A.B., Tschampel, S.M., Gonzalez-Outeirino, J., Daniels, C.R., Foley, B.L., and Woods, R.J. (2008). GLYCAM06: a generalizable biomolecular force field. Carbohydrates. J. Comput. Chem. 29: 622-655.

Koehler, L., Ruiz-Gómez, G., Balamurugan, K., Rother, S., Freyse, J., Möller, S., Schnabelrauch, M., Köhling, S., Djordjevic, S., Scharnweber, D., et al. (2019). Dual action of sulfated hyaluronan on angiogenic processes in relation to vascular endothelial growth factor-A. Sci. Rep. 9: 18143.

Kohler, N. and Lipton, A. (1974). Platelets as a source of fibroblast growth-promoting activity. Exp. Cell Res. 87: 297-301.

Köhling, S., Kuenze, G., Lemmnitzer, K., Bermudez, M., Wolber, G., Schiller, J., Huster, D., and Rademann, J. (2016).

Chemoenzymatic synthesis of nonasulfated tetrahyaluronan with a paramagnetic tag for studying its complex with interleukin-10. Chem. Eur J. 22: 5563-5574.

Köhling, S., Blaszkiewicz, J., Ruiz-Gómez, G., Fernández-Bachiller, M.I., Lemmnitzer, K., Panitz, N., Beck-Sickinger, A., Schiller, J., Pisabarro, M.T., and Rademann, J. (2019). Syntheses of defined sulfated oligohyaluronans reveal structural effects, diversity and thermodynamics of GAG-protein binding. Chem. Sci. 10: 866-878. 
Kuai, J., Mosyak, L., Brooks, J., Cain, M., Carven, G.J., Ogawa, S., Ishino, T., Tam, M., Lavallie, E.R., Yang, Z., et al. (2015). Characterization of binding mode of action of a blocking anti-platelet-derived growth factor (PDGF)-B monoclonal antibody, MOR8457, reveals conformational flexibility and avidity needed for PDGF-BB to bind PDGF receptor- $\beta$. Biochemistry 54: 1918-1929.

Mamer, S.B., Chen, S., Weddell, J.C., Palasz, A., Wittenkeller, A., Kumar, M., and Imoukhuede, P.I. (2017). Discovery of highaffinity PDGF-VEGFR interactions: redefining RTK dynamics. Sci. Rep. 7: 16439.

Miller, B.R., III., McGee, T.D., Jr., Swails, J.M., Homeyer, N., Gohlke, H., and Roitberg, A.E. (2012). MMPBSA.py: an efficient program for end-state free energy calculations. J. Chem. Theor. Comput. 8: 3314-3321.

Morris, G.M., Goodsell, D.S., Halliday, R.S., Huey, R., Hart, W.E., Belew, R.K., and Olson, A.J. (1998). Automated docking using a Lamarckian genetic algorithm and an empirical binding free energy function. J. Comput. Chem. 19: 1639-1662.

Necas, J., Bartosikova, L., Brauner, P., and Kolar, J. (2008). Hyaluronic acid (hyaluronan): a review. Vet. Med. 53: 397-411.

Ostman, A. (2004). PDGF receptors-mediators of autocrine tumor growth and regulators of tumor vasculature and stroma. Cytokine Growth Factor Rev. 15: 275-286.

OriginLab, Northampton, MA. (2019), http://www.originlab.com.

Ross, R., Glomset, J., Kariya, B., and Harker, L. (1974). A plateletdependent serum factor that stimulates the proliferation of arterial smooth muscle cells in vitro. Proc. Natl. Acad. Sci. U. S. A 71: $1207-1210$.

Rostovtsev, V.V., Green, L.G., Fokin, V.V., and Sharpless, K.B. (2002). A stepwise huisgen cycloaddition process: copper(I)-catalyzed regioselective "ligation" of azides and terminal alkynes. Angew. Chem. Int. Ed. 41: 2596-2599.

Rother, S., Samsonov, S.A., Moeller, S., Schnabelrauch, M., Rademann, J., Blaszkiewicz, J., Kohling, S., Waltenberger, J., Pisabarro, M.T., Scharnweber, D., et al. (2017). Sulfated hyaluronan alters endothelial cell activation in vitro by controlling the biological activity of the angiogenic factors vascular endothelial growth factor-A and tissue inhibitor of metalloproteinase-3. ACS Appl. Mater. Interfaces 9: 9539-9550.

Rother, S., Ruiz-Gómez, G., Balamurugan, K., Koehler, L., Fiebig, K.M., Galiazzo, V.D., Hempel, U., Moeller, S., Schnabelrauch, M., Waltenberger, J., et al. (2021). Hyaluronan/collagen hydrogels with sulfated glycosaminoglycans maintain $\mathrm{VEGF}_{165}$ activity and fine-tune endothelial cell response. ACS Appl. Bio Mater. 4: 494-506.

Salbach-Hirsch, J., Kraemer, J., Rauner, M., Samsonov, S.A., Pisabarro, M.T., Moeller, S., Schnabelrauch, M., Scharnweber, D., Hofbauer, L.C., and Hintze, V. (2013). The promotion of osteoclastogenesis by sulfated hyaluronan through interference with osteoprotegerin and receptor activator of NF-KB ligand/osteoprotegerin complex formation. Biomaterials 34: 7653-7661.

Samsonov, S. and Pisabarro, M.T. (2016). Computational analysis of interactions in structurally available proteinglycosaminoglycan complexes. Glycobiology 26: 850-861.

Scharnweber, D., Hübner, L., Rother, S., Hempel, U., Anderegg, U., Samsonov, S.A., Pisabarro, M.T., Hofbauer, L., Schnabelrauch, M., Franz, S., et al. (2015). Glycosaminoglycan derivatives: promising candidates for the design of functional biomaterials. J. Mater. Sci. Mater. Med. 26: 232.
Schlessinger, J. (2000). Cell signaling by receptor tyrosine kinases. Cell 103: 211-225.

Schrödinger, L. (2009-2015). The PyMOL molecular graphics system, Version 1.8.

Shim, H.-R.A., Liu, H., Focia, P.J., Chen, X.P., Lin, P.C., and He, X. (2010). Structures of a platelet-derived growth factor/ propeptide complex and a platelet-derived growth factor/ receptor complex. Proc. Natl. Acad. Sci. U.S.A. 107: 11307-11312.

Siegfried, G., Basak, A., Prichett-Pejic, W., Scamuffa, N., Ma, L., Benjannet, S., Veinot, J.P., Calvo, F., Seidah, N., and Khatib, A.M. (2005). Regulation of the stepwise proteolytic cleavage and secretion of PDGF-B by the pro-protein convertases. Oncogene 24: 6925-6935.

Su, W., Liu, G., Liu, X., Zhou, Y., Sun, Q., Zhen, G., Wang, X., Hu, Y., Gao, P., Demehri, S., et al. (2020). Angiogenesis stimulated by elevated PDGF-BB in subchondral bone contributes to osteoarthritis development. JCI Insight 5: 2379-3708.

Thönes, S., Rother, S., Wippold, T., Blaszkiewicz, J., Balamurugan, K., Moeller, S., Ruiz-Gómez, G., Schnabelrauch, M., Scharnweber, D., Saalbach, A., et al. (2019). Hyaluronan/collagen hydrogels containing sulfated hyaluronan improve wound healing by sustained release of heparin-binding EGF-like growth factor. Acta Biomater. 86: 135-147.

Tornoe, C.W., Christensen, C., and Meldal, M. (2002). Peptidotriazoles on solid phase: [1,2,3]-triazoles by regiospecific copper(i)-catalyzed 1,3-dipolar cycloadditions of terminal alkynes to azides. J. Org. Chem. 67: 3057-3064.

Vallet, S.D., Clerc, O., and Ricard-Blum, S. (2020). Glycosaminoglycanprotein interactions: the first draft of the glycosaminoglycan interactome. J. Histochem. Cytochem. 69(2): 93-104.

van der Smissen, A., Samsonov, S.A., Hintze, V., Scharnweber, D., Moeller, S., Schnabelrauch, M., Pisabarro, M.T., and Anderegg, U. (2013). Artificial extracellular matrix composed of collagen I and high-sulfated hyaluronan interferes with TGF $\beta 1$ signaling and prevents TGF $\beta 1$ induced myofibroblast differentiation. Acta Biomater. 9: 7775-7786.

Wang, J.M., Morin, P., Wang, W., and Kollman, P.A. (2001). Use of MM-PBSA in reproducing the binding free energies to HIV-1 RT of TIBO derivatives and predicting the binding mode to HIV-1 RT of efavirenz by docking and MM-PBSA. J. Am. Chem. Soc. 123: 5221-5230.

Wang, X., Wu, X., Zhanga, A., Wanga, S., Hu, C., Chena, W., Shena, Y., Tan, R., Sun, Y., and Xu, Q. (2016). Targeting the PDGF-B/PDGFR$\beta$ interface with destruxin A5 to selectively block PDGF-BB/ PDGFR- $\beta \beta$ signaling and attenuate liver fibrosis. EBioMedicine 7: 146-156.

Waterhouse, A., Bertoni, M., Bienert, S., Studer, G., Tauriello, G., Gumienny, R., Heer, F.T., de Beer, T.A.P., Rempfer, C., Bordoli, L., et al. (2018). SWISS-MODEL: homology modelling of protein structures and complexes. Nucleic Acids Res. 46: W296-W303.

Xing, F., Zhou, C., Hui, D., Du, C., Wu, L., Wang, L., Wang, W., Pu, X., Gu, L., Liu, L., et al. (2020). Hyaluronic acid as a bioactive component for bone tissue regeneration: fabrication, modification, properties, and biological functions. Nanotechnol. Rev. 9: 1059-1079.

Xu, D. and Esko, J.D. (2014). Demystifying heparan sulfate-protein interactions. Annu. Rev. Biochem. 83: 129-157.

Supplementary Material: The online version of this article offers supplementary material (https://doi.org/10.1515/hsz-2021-0173). 